\title{
Analysis and Performance Evaluation of Bluetooth Low Energy Piconet Network
}

\author{
Ziyad Khalaf Farej, Aydin M. Saeed \\ Technical Engineering College, Northern Technical University, Mosul, Iraq \\ Email:drziyad.farej@ntu.edu.iq, aydinmsa@yahoo.com
}

How to cite this paper: Farej, Z.K. and Saeed, A.M. (2020) Analysis and Performance Evaluation of Bluetooth Low Energy Piconet Network. Open Access Library Journal, 7: e6814.

https://doi.org/10.4236/oalib.1106814

Received: September 12, 2020

Accepted: October 24, 2020

Published: October 27, 2020

Copyright $\odot 2020$ by author(s) and Open Access Library Inc.

This work is licensed under the Creative Commons Attribution International License (CC BY 4.0).

http://creativecommons.org/licenses/by/4.0/

\section{(c) (i) Open Access}

\begin{abstract}
This paper presents the performance analysis of Bluetooth Low Energy (BLE) IEEE 802.15.1 standard in the piconet topology networks. The performance of the BLE network is analyzed in terms of connection interval (CI) and the number of slaves $\left(\mathrm{N}_{\mathrm{S}}\right)$ by using OMNET++ (v4.6) simulator. In the performance investigation process Data Length Extension (DLE) features is used with an application payload size of 60 bytes in order to achieve the best performance in a BLE (P2P) network. The modeled networks and due to the nature of short-range BLE wireless communication show a good matching between analytical and simulation results. For Single-Slave (or one like) piconet the obtained maximum simulation throughput is $478.7 \mathrm{kbps}$ when a connection interval of (7.5) $\mathrm{ms}$ is used.
\end{abstract}

\section{Subject Areas}

Wireless Networks, Wireless Communications, Computer Engineering

\section{Keywords}

Bluetooth Low Energy (BLE), Piconet, Data Length Extension (DLE), Connection Interval (CI), Number of Slaves $\left(\mathrm{N}_{\mathrm{S}}\right)$

\section{Introduction}

Bluetooth Low Energy (BLE) is a short-range wireless communication and devoted to low power and low-cost transmissions, technology developed by the Bluetooth Special Interest Group (SIG). The aim of this group is to make this technology the most suitable substitution to a large number of already present and widely spread wireless technologies such as (Classic (3.0, basic or enhanced data rate) Bluetooth, IEEE 802.11b and ZigBee) [1] [2]. BLE was first released as Bluetooth 4.0 in 2010, and it has fabulous attention as one of the basic technolo- 
gies for the Internet of Things (IoT) and is used for many applications [3]. In 2014, the IoT functionality is greatly enhanced through BLE 4.2 application which allows devices to directly transmit data over the Internet [4]. As well as a high number of wireless technologies use the ISM band for data communication, this leads to more congestion and noise, however, the wireless BLE uses a frequency-hopping method (for both its advertising and data channels) to prevent interference during communication. The classic Bluetooth network has 79 channels with $1 \mathrm{MHz}$ width each, while the BLE use 40 channels in the ISM (2.4 $\mathrm{GHz}$ ) band each with a width of $2 \mathrm{MHz}$ Of these 40 channels, three channels (namely channels 37, 38, and 39 are used for advertising purposes) and the remaining 37 channels are responsible for the data transmission. The device discovery operation is optimized, through the developing of a brief state-machine which also includes the energy-saving features [5] [6]. The new characteristic that is known as (LE Data Packet Length Extension), is introduced in BLE4.2. This characteristic allows BLE4.2 device to Data Length Extension (DLE) Payload in a Link Layer (LL) packet from 27 up to 251 bytes, so a data of 251 bytes can now be sent instead of 27 bytes in the payload. As a result, more data can be transmitted with less number of vacuums between the packets known as (inter-frame space (TIFS $=150 \mu \mathrm{s})$ ). So this feature enhances the speed of data transmission [7]. Although Bluetooth wireless personal area network (WPAN) is a technology developed for different uses by both personal computers and mobile devices (like telephones and other wireless devices), it is predicted that some interference may occur in the same nature when two of these networks work concurrently and in the same range [8]. The BLE is widely applied in different fields. The body area network (BAN) applications focused primarily in networks with a point-to-point (single slave) with relatively low throughput. For example in the medical field, the BAN devices are equipped with this technology for e-health care such as (an Inertial Measurement Units (IMU) sensor that used for early diagnosis of blood pressure, blood glucose level, electroencephalography (EEG), heart rate sensor electrocardiography (ECG), electromyography (EMG) for Control prostheses and as well as used to monitor breathing), it is also used among others (voice communications, smart houses, security systems, in homes with private health care environments and automotive) [1] [9]. In this paper, the DLE feature of the BLE and its effect on the throughput performance of the (single and multiple slaves piconet) networks is analyzed and investigated. As well as the effect of other parameters (Maximum Transmission Unit (MTU) and packet size per connection interval), which commonly vary according to the designed equipment and the required application, is also considered to improve and optimize network performance.

\section{Related Works}

Although the development of the BLE system (at Physical and MAC layers) is recently focusing on support of various IoT applications (like smart home applications, E-health), there is a wide range of working fields for servicing the 
academic community. The effect of the bit error rate (BER) to the maximum throughput value is taken into account to evaluate the network performance by [10]. The BLE performance in exchanging data between two nodes (master and slave) is investigated. The achieved maximum throughput and how to improve its value is also considered by increasing packet size through the Maximum Transmission Unit (MTU) in the application payload [11] [12]. Reference [13] presented an analytical method to estimate the BLE 4.2 maximum throughput of a peer-to-peer (P2P) link at the application layer. Their results show that the Bit Error Rate (BER) is zero for error-free communication and for 20 bytes payload the maximum throughput value is $236.7 \mathrm{kbps}$. Also this value corresponds to the most suitable value of the standard BLE protocol. Wha et al. [3] studied the BLE for IoT applications, they used multi-packet criteria to transfer packets between master and slaves with a high probability of data channel accessing. According to their proposed mechanism a message-based alternation, instead of the packet, during the connection event is adapted. To avoid bad quality link and keep transmission continuous, they also suggested the channel-hopping technology among the available BLE channels rather than sleeping, as a result system performance in terms of throughput and delay is enhanced, however there was a slight increase in power consumption. For Body Area Network (BAN) and Wireless Sensor Network (WSN) applications, the communication between nodes (P2P link) and the effect of increasing and decreasing the number of slaves on a Multi-Slave BLE system performance along with network energy consumption are evaluated by [9] [14].

\section{Overview of the Bluetooth Low Energy Protocol Stack}

The BLE protocol (standard IEEE 802.15.1) which supports low-energy communications among devices, contains three main parts. These parts as shown in Figure 1 are the Controller, Host and Application (App) layers at the Physical Layer (PHY).

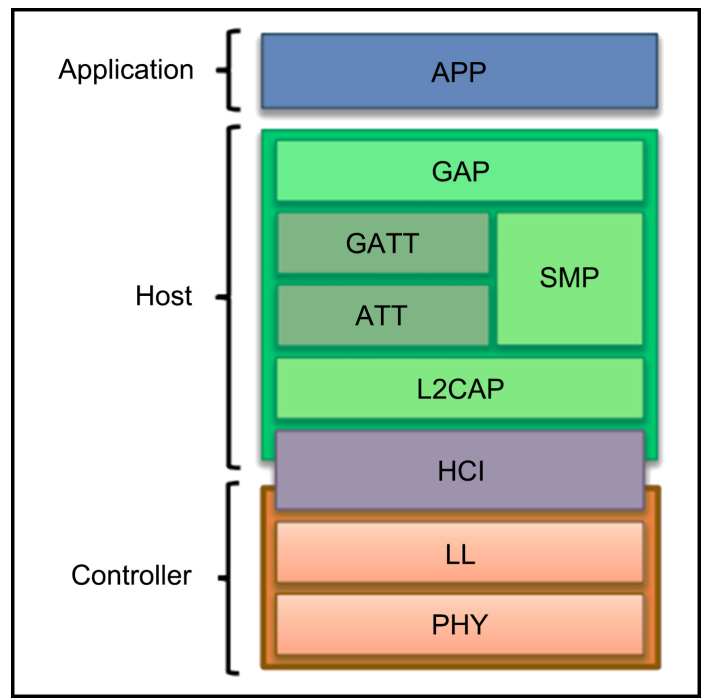

Figure 1. BLE protocol stack. 
BLE operates in the $2.4 \mathrm{GHz}$ Industrial Scientific Medical (ISM) band at a data rate of $2 \mathrm{Mbit} / \mathrm{s}$. As shown in Figure 2, its band is divided into 40 Radio Frequency (RF) channels ( 3 of them for advertising and 37 for bi-directional data exchange among two devices). To avoid interference in the same radio band with other wireless communications Adaptive Frequency Hopping (AFH) is used by the data channels. The easy to implement Gaussian Frequency Shift Keying (GFSK) modulation is used by all channels with modulation index of 0.5 for low peak energy consumption [4].

The Link Layer LL manages the radio's link-state and defines the Frequency Hopping transmissions that can be generated among BLE devices. These transmissions include the broadcast of advertising packets via the advertising channels. It also determines the operating function for each device (such as master, slave, advertiser and scanner). An initiator should discover an advertiser before it sends a message for connection request so that a communication between two devices can be established across the data channels. For this connection, a randomly generated (called access address) packet with a length of 32 bits is used. At the Link Layer BLE defines two (master and slave) device roles to establish this connection. The standard interface protocol that makes communication between Controller and Host, is the Host Controller Interface (HCI).

The Host which is the core of the BLE protocol stack handles the communication among the user application and the hardware. So it defines a set of commands and algorithms to transfer actual data into data packets sent to the Host layer through the serial port and vice versa. As in Bluetooth classic, the Logical Link Control and Adaptation Protocol (L2CAP) forms the basis of the BLE protocol in a simplified and improved way where it takes the data packets from the data processor in the LL and encapsulates them in the packet format of the BLE protocol and according to the data used in the higher layers (SMP, ATT), such process is called encapsulation, while in the reverse direction packets are fragmented in a process called fragmentation. The Security Manager Protocol (SMP) supports secure identity and data encryption through its major distribution features. The host also operates on the application-processor to interact and implement features at the application level by its ATT, GAP and GATT Protocols. The server and client functions are defined by the Attribute Protocol (ATT) to

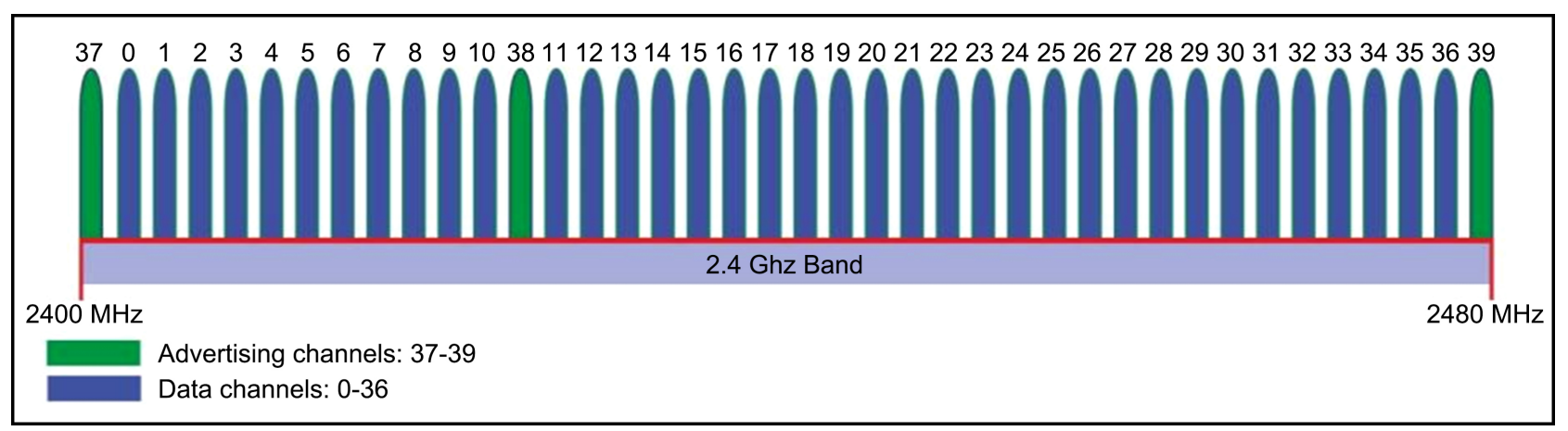

Figure 2. BLE frequency channels. 
manage the connection among two devices. The discovery services and management of characteristic interchange from one device to another are accomplished by the Generic ATT (GATT) through the definition of framework which utilizes the ATT Protocol. The Generic Access Profile (GAP) defines the generic procedures for discovery, modes, link management and services [15] [16] [17]. The data packet format at all layer for the BLE protocol stack is shown in Figure 3.

\section{Bluetooth Low Energy Theoretical Performance}

\section{Throughput Performance Analysis of Piconet Network}

In this paper, the data transfer process is analyzed with the help of notifications from an ATT (master) to an ATT (slave) device, so that the message packets are transmitted by the slave without prior receipt of an ACK, and the master does not need to validate receipt of the data either. As in normal case, this performance is analyzed under the assumption that the connection between the master and the slaves is completely within the transmission range of each other.

As shown in Figure 4, in the BLE connection the time in which data packets are transferred among master and slave is called Connection Event (ConnEvent). This event consists of a number of Round trip times in connection with the move of data packets and their ACKs, while the remaining time is called Radio Idle when the communication is off. The Anchor point is the start of a Con$n E v e n t$, where the master starts to send packets to the slave. Time inter-frame space $\mathrm{T}_{\mathrm{IFS}}$ is guard times among packets. Connection Interval (ConnInterval) is the time between the beginnings of two successive ConnEvents. This time, (which represents the total time of ConnEvent and Radio Idle), is a multiple of $1.25 \mathrm{~ms}$ in the range of $7.5-4000 \mathrm{~ms}$ [4].

In BLE the overall packet size is $1+4+(2 \sim 257)+3$ bytes (Preamble + Access Address + PDU + CRC), as it is previously shown in Figure 3 . All the advertising and data packets have the same structure with the distinction being in the PDU field only. The transmission times for the master $T_{S}$ and slave $T_{M}$ packets are given by:

\begin{tabular}{|c|c|c|c|c|c|c|c|c|}
\hline Preamble & Access Address & \multicolumn{6}{|c|}{ PDU (2 - 257 bytes) } & CRC \\
\hline \multirow{5}{*}{1 byte } & \multirow{5}{*}{4 bytes } & $\begin{array}{l}\text { LL } \\
\text { Header }\end{array}$ & \multicolumn{4}{|c|}{ Payload ( 0 - 251 bytes) } & \multirow{2}{*}{$\begin{array}{c}\text { MIC } \\
\text { (Optional) }\end{array}$} & \multirow{5}{*}{3 bytes } \\
\hline & & \multirow{4}{*}{2 bytes } & $\begin{array}{l}\text { L2CAP } \\
\text { Header }\end{array}$ & \multicolumn{3}{|c|}{ ATT Data ( 0 - 247 bytes) } & & \\
\hline & & & \multirow{3}{*}{4 bytes } & ATT $\mathrm{H}$ & ader & ATT Payload & \multirow{3}{*}{4 bytes } & \\
\hline & & & & Op Code & $\begin{array}{c}\text { ATT } \\
\text { Handle }\end{array}$ & \multirow[t]{2}{*}{$\leq 244$ bytes } & & \\
\hline & & & & 1 byte & 2 bytes & & & \\
\hline & \multicolumn{2}{|c|}{ Physical/Link layer } & & \multicolumn{2}{|c|}{ L2CAP layer } & \multirow{2}{*}{\multicolumn{2}{|c|}{ ATT layer }} & \\
\hline & \multicolumn{2}{|c|}{ Data channel PDU } & & \multicolumn{2}{|c|}{ GATT layer } & & & \\
\hline
\end{tabular}

Figure 3. BLE data packet format. 


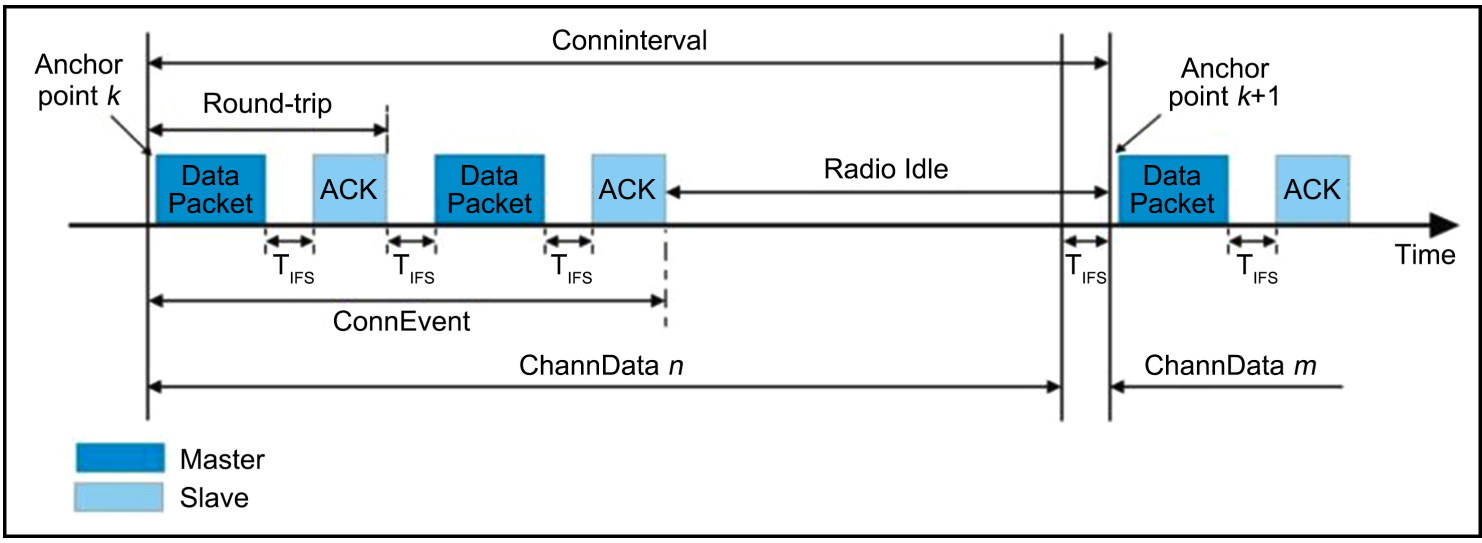

Figure 4. BLE connection between a master and a slave.

$$
\begin{gathered}
\mathrm{T}_{\mathrm{S}}=\mathrm{T}_{\mathrm{Pr}}+\mathrm{T}_{\mathrm{aa}}+\mathrm{T}_{\text {llh }}+\mathrm{T}_{\mathrm{CRC}}+\mathrm{T}_{\mathrm{PDU}}+\mathrm{T}_{\mathrm{op}}+\mathrm{T}_{\mathrm{ATTh}}+\mathrm{T}_{\mathrm{L} 2 \mathrm{CAPh}} \\
\mathrm{T}_{\mathrm{M}}=\mathrm{T}_{\mathrm{Pr}}+\mathrm{T}_{\mathrm{aa}}+\mathrm{T}_{\mathrm{llh}}+\mathrm{T}_{\mathrm{CRC}}
\end{gathered}
$$

where $\mathrm{T}_{\mathrm{Pr}}, \mathrm{T}_{\mathrm{aa}}, \mathrm{T}_{\text {llh }}, \mathrm{T}_{\mathrm{CRC}}, \mathrm{T}_{\mathrm{op}}, \mathrm{T}_{\mathrm{ATTh}}, \mathrm{T}_{\mathrm{L} 2 \mathrm{CAPh}}$ and $\mathrm{T}_{\mathrm{PDU}}$ denotes the transmission times of the packets fields Preamble, Access Address, Link layer header, CRC, ATT opcode, ATT handle, L2CAP header and PDU respectively.

Taking into consideration that the BLE data rate is $1 \mathrm{Mbit} / \mathrm{sec}$, the time for the master to send control information to slave is extremely small (so that it can be neglected), hence, the payload for the link level is empty. Also based on the assumption that the master sends to the slave a packet of application payload size of 60 bytes (in the ATT value field), then from Equations (1) and (2), $\mathrm{T}_{\mathrm{M}}(=80$ $\mu \mathrm{s})$ and $\mathrm{T}_{\mathrm{S}}(=616 \mu \mathrm{s})$ can be calculated. The overall time for the interchange of packets between the master and the slave $\mathrm{T}_{\mathrm{MS}}$ is equal to $996 \mu$ s (with the guard times among packets is $\mathrm{T}_{\mathrm{IFS}}=150 \mu \mathrm{s}$ ) as described below in Equation (3). So when the BLE devices transmit continuously a maximum throughput of 481.9 kbps $\left(=\frac{60 \times 8}{996}\right)$ on each orientation can be acquired.

$$
\mathrm{T}_{\mathrm{MS}}=\mathrm{T}_{\mathrm{S}}+\mathrm{T}_{\mathrm{IFS}}+\mathrm{T}_{\mathrm{M}}+\mathrm{T}_{\mathrm{IFS}}
$$

When the master is serving one slave, the maximum available throughput $\mathrm{TH}_{\text {maxs-s }}$ of Single-Slave piconet for BLE model can be given by:

$$
\mathrm{Th}_{\text {maxs-s }}=\frac{\mathrm{N}_{\mathrm{p}} \times \mathrm{L}_{\text {data }} \times 8 \text { bits }}{\mathrm{T}_{\mathrm{CI}}}
$$

where $\mathrm{N}_{\mathrm{p}}$ is the number of the packet per each connection event, $\mathrm{L}_{\text {data }}$ is the application layer payload length in bytes and $\mathrm{T}_{\mathrm{CI}}$ is the time connection interval.

In order to calculate the maximum throughput $\mathrm{TH}_{\text {maxM-s }}$ in the network when the master is sending 60 bytes of data to a number of slaves $\mathrm{N}_{\mathrm{S}}$ over the BLE network, it is needed to specify the total time $\mathrm{T}_{\text {Total }}$ wanted for a slave to send all its number of round-trip $\mathrm{N}_{\text {round-trip }}$ in a connection event. This period, in effect, is the result of $\mathrm{N}_{\text {round-trip }}$ by the time needed for packet exchange between master and slave $\mathrm{T}_{\mathrm{MS}}$. Now if we assume that all slaves share equal time, then the supported number of slaves $\mathrm{N}_{\mathrm{S}}$ can be obtained as follows: 


$$
\mathrm{N}_{\mathrm{S}}=\frac{\mathrm{T}_{\mathrm{CI}}}{\mathrm{T}_{\text {Total }}}=\frac{\mathrm{T}_{\mathrm{CI}}}{\mathrm{N}_{\text {round-trip }} \times \mathrm{T}_{\mathrm{MS}}}
$$

Based on the assumption that the SlaveLatency is equal to zero for Synchronous Connection Oriented (SCO) system mode operation, then combining Equations (4) and (5), the maximum throughput $\mathrm{TH}_{\operatorname{maxM}-\mathrm{s}}$ of the Multi-Slave piconet network can be given according to the follows equation:

$$
\mathrm{TH}_{\text {maxM-S }}=\frac{\mathrm{N}_{\mathrm{p}} \times \mathrm{L}_{\text {data }} \times 8 \text { bits }}{\mathrm{N}_{\mathrm{S}} \times \mathrm{N}_{\text {round-trip }} \times \mathrm{T}_{\mathrm{MS}}}
$$

\section{BLE Network Modeling and Assumption}

The basic BLE network (Single-Slave piconet) is composed of a master and a slave. Although each device has the ability to perform master or slave roles, the master cannot contact more than one slave at the same time. The Multi-Slave piconet Topology (one master and seven slaves) network as shown in Figure 5 is designed and modeled by using the OMNeT++ simulation tool (v4.6) [18].

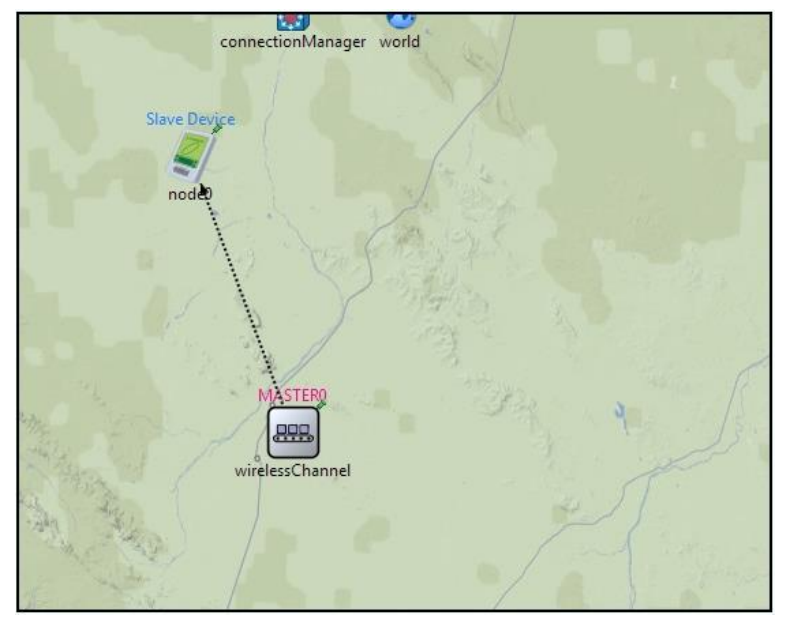

(a)

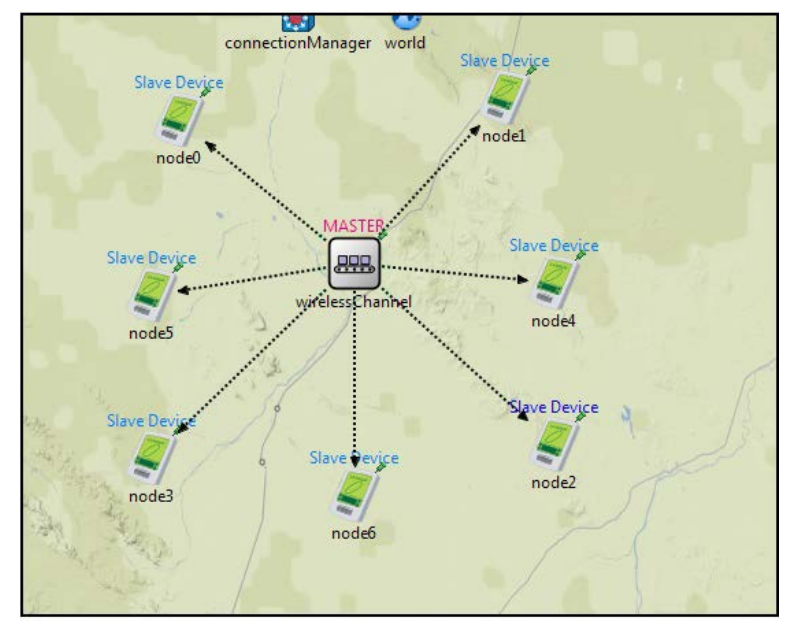

(b)

Figure 5. BLE network design. (a) Single-Slavepiconet; (b) Multi-Slave piconet. 
The simulator is based on $\mathrm{C}++$ code to model communication networks and its basic tool MiXiM framework (v2.3) is used to provide details for wireless channel network. This simulator is excuted to simulate the modeled networks.

At the MAC layer, the set of the LL is extended and DLE is enabled to increase the data rate and enable multiple channel communication within the BLE specification. The networks are modeled under the following assumptions:

1) The modeled simulation area is $100 \mathrm{~m} \times 100 \mathrm{~m}$.

2) All nodes are within the transmission range of each other.

3) DLE feature is enabled.

4) The slaves have equal distances and all are one hop from master.

5) The connection slave latency is equal to zero.

6) The size of application payload is 60 bytes.

7) It is assumed that there is no multipath effect problem, and simulated according to the BLE standard link with simulation parameters summarized in Table 1.

\section{Simulation Results and Analysis}

The modeled BLE based Multi-Slave piconet topology and Single-Slave piconets are simulated under the tabulated link simulation parameter set. The best performance of these networks is investigated and optimized by changing a number of performance parameters: connection interval, number of slaves and number of links, for application payload with packet size of 60 bytes.

\subsection{Single-Slave Piconet}

The throughput performance for different connection interval between master and one slave for this topology network is investigated with different payload sizes of values $(3,6,8$ and 12) packets with 60 bytes each. The theoretical and simulation results for the throughput decrease gradually with increasing connection interval as shown in Figure 6. High consistent between theoretical and simulation results is seen as well as (for 12 packets payload) maximum and minimum throughput value of (767.8) kbps and (1.5) kbps at connection intervals of (7.5) $\mathrm{ms}$ and (4000) ms respectively.

Table 1. The simulation parameters of the BLE.

\begin{tabular}{cc}
\hline Parameter & Value \\
\hline Buffer size (kbits) & 1024 \\
Data rate (kbps) & 1000 \\
AdvInterval (ms) & 25 \\
ScanInterval (ms) & 25 \\
ConnInterval (ms) & 10 \\
ConnSlaveLatency & 0 \\
Payload size (bytes) & 60 \\
Mobility type & Stationary Mobile \\
Simulation time (min) & 1 \\
\hline
\end{tabular}




\subsection{Multi-Slaves Piconet}

The results show that the throughput value decreases gradually with the increasing number of slaves as shown in Figure 7. This is due to the fact that the data in the master will be distributed over all the slaves in the network. For theoretical and simulation results, the maximum throughput values are (481.8 kbps and $479.7 \mathrm{kbps}$ ) in case of one salve and minimum throughput values are (68.8 kbps and $66.2 \mathrm{kbps}$ ) in case of seven slaves respectively. The approximation in the results which considered the SlaveLatency equals to zero, is the reason behind the small difference between theoretical and simulation results.

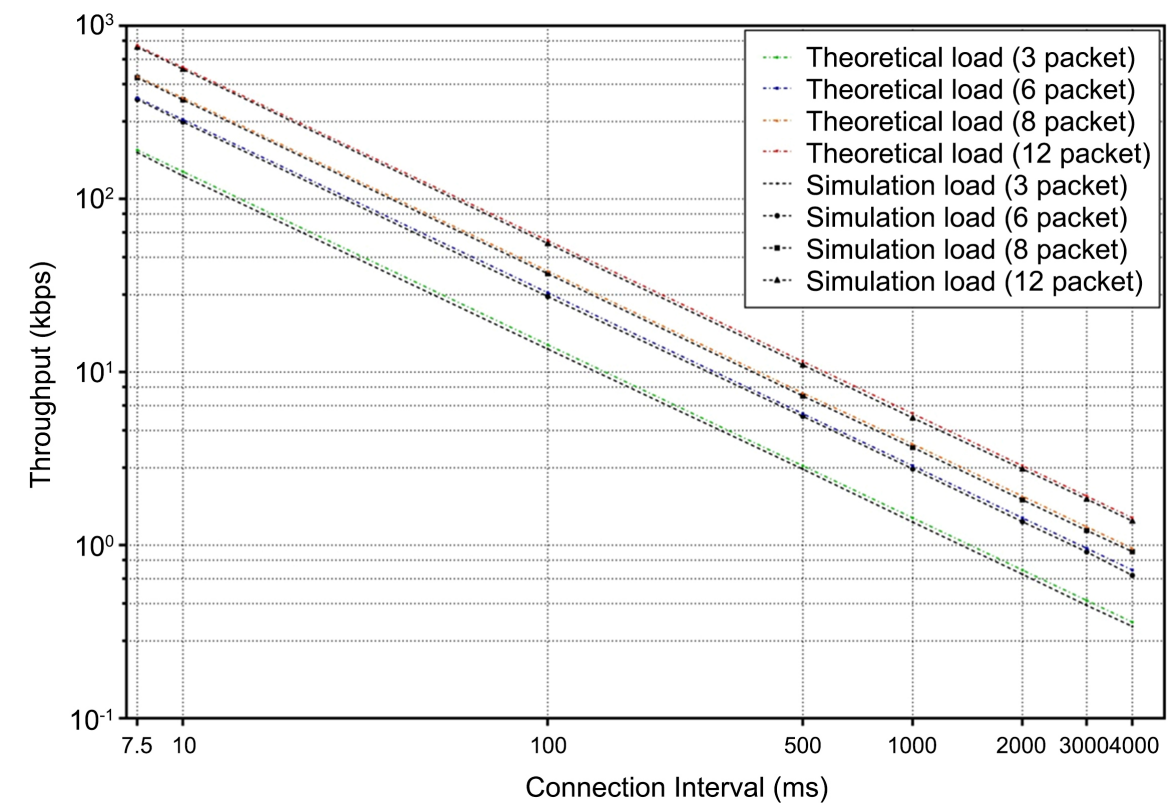

Figure 6. Throughput for Single-Slave piconet.

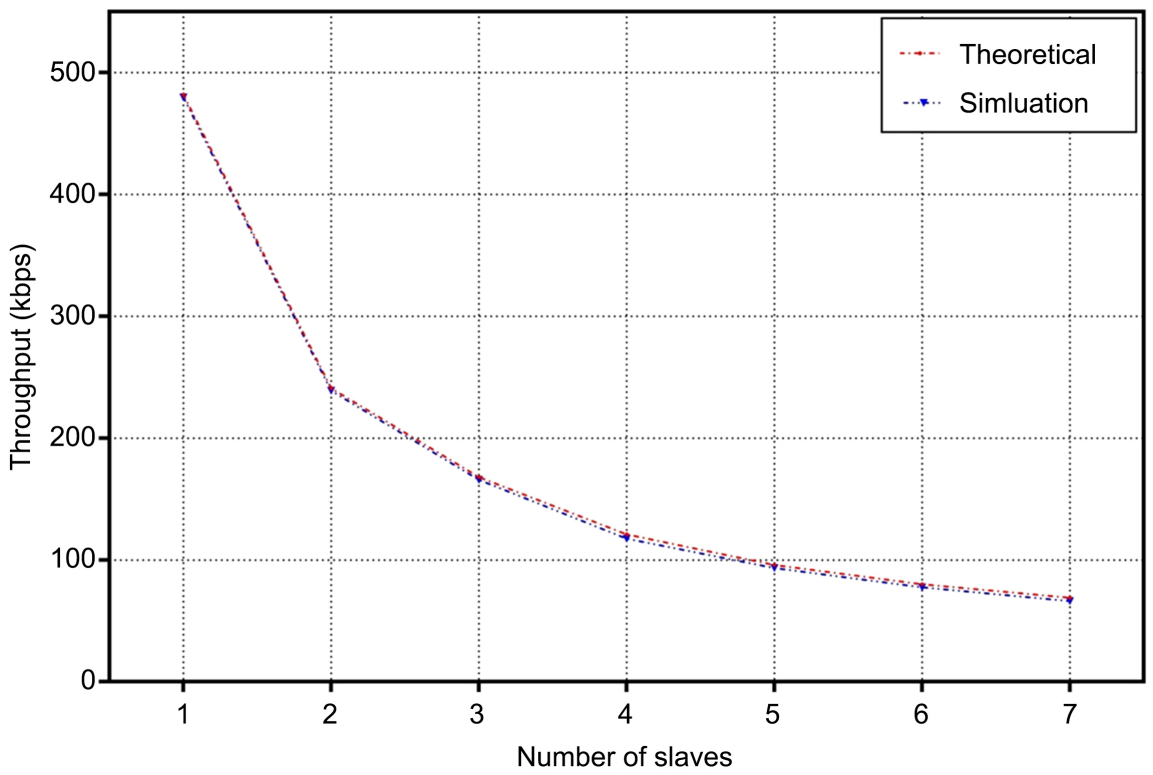

Figure 7. Throughput for Multi-Slaves Piconet. 


\section{Conclusion}

The throughput performance of the BLE based single-slave and multiple-slaves piconet topologies are analyzed by changing the parameters connection interval and the number of slaves. After you enable the data Length Extension (DLE) Property, gives an increase in the throughput for modeled piconet network. For 12 packets with 60 bytes application payload (and at $7.5 \mathrm{~ms}$ connection interval), the simulation results of the Single-Slave BLE piconet shows a maximum throughput value of (755.8) kbps which is very close to the analytical (767.8) kbps value. Also, it is found that increasing the number of slaves decreases the maximum simulated throughput to (66.2) kbps. Also the close consistent between theoretical and simulation results is due to the short-range and deterministic channel access (TDMA) mechanism for this type of wireless communication standards.

\section{Acknowledgements}

This work was supported by the Technical Engineering College-Mosul, Northern Technical University (NTU) and my supervisor Dr. Ziyad K. Farej.

\section{Conflicts of Interest}

The authors declare no conflicts of interest regarding the publication of this paper.

\section{References}

[1] Tosi, J., Taffoni, F., Santacatterina, M., Sannino, R. and Formica, D. (2017) Performance Evaluation of Bluetooth Low Energy: A Systematic Review. Sensors (Switzerland), 17, 2898. https://doi.org/10.3390/s17122898

[2] Farej, Z.K. and Abdul-hameed, A.M. (2017) Performance Analysis of IEEE 802.15.4 Based Sensor Networks for Large Scale Tree Topology. Circulation in Computer Science, 2, 9-13. https://doi.org/10.22632/ccs-2017-252-41

[3] Jeon, W.S. and Jeong, D.G. (2017) Enhanced Channel Access for Connection State of Bluetooth Low Energy Networks. IEEE Transactions on Vehicular Technology, 9 , 8469-8481. https://doi.org/10.1109/TVT.2017.2675915

[4] Bluetooth Special Interest Group (2014) Specification of the Bluetooth System Covered Core Package Version 4.2. History, 2272.

[5] Cho, K., Park, W., Hong, M., Park, G., Cho, W., Seo, J. and Han, K. (2015) Analysis of Latency Performance of Bluetooth Low Energy (BLE) Networks. Sensors (Switzerland), 15, 59-78. https://doi.org/10.3390/s150100059

[6] Al Kalaa, M.O. and Refai, H.H. (2015) Selection Probability of Data Channels in Bluetooth Low Energy. 2015 International Wireless Communications and Mobile Computing Conference (IWCMC), Dubrovnik, 24-28 August 2015, 148-152. https://doi.org/10.1109/IWCMC.2015.7289073

[7] ManasMarawaha, J.D., Jha, P. and Razdan, R. (2018) Performance Evaluation of Bluetooth Low Energy Communication. Journal of Information Sciences and Computing Technologies, 7, 718-725.

[8] Okhaifor, J., Emagbetere, J. and Edeko, F. (2014) Interference Effects of Blue Tooth 
on WLAN Performance. Nigerian Journal of Technology, 34, 177-183. https://doi.org/10.4314/njt.v34i1.22

[9] Afonso, J.A., Maio, A.J.F. and Simoes, R. (2016) Performance Evaluation of Bluetooth Low Energy for High Data Rate Body Area Networks. Wireless Personal Communications, 90, 121-141. https://doi.org/10.1007/s11277-016-3335-4

[10] Gomez, C., Demirkol, I. and Paradells, J. (2011) Modeling the Maximum Throughput of Bluetooth Low Energy in an Error-Prone Link. IEEE Communications Letters, 15, 1187-1189. https://doi.org/10.1109/LCOMM.2011.092011.111314

[11] Mikhaylov, K. and Tervonen, J. (2013) Multihop Data Transfer Service for Bluetooth Low Energy. 13th International Conference on ITS Telecommunications (ITST), Tampere, 5-7 November 2013, 319-324.

https://doi.org/10.1109/ITST.2013.6685566

[12] Todtenberg, N. and Kraemer, R. (2019) A Survey on Bluetooth Multi-Hop Networks. Ad Hoc Networks, 93, Article ID: 101922.

https://doi.org/10.1016/j.adhoc.2019.101922

[13] Dian, F.J., Yousefi, A. and Lim, S. (2019) A Practical Study on Bluetooth Low Energy (BLE) Throughput. 9th Annual Information Technology, Electronics and Mobile Communication Conference, Vancouver, 1-3 November 2018, 768-771. https://doi.org/10.1109/IEMCON.2018.8614763

[14] Mikhaylov, K. (2014) Simulation of Network-Level Performance for Bluetooth Low Energy. IEEE 25th Annual International Symposium on Personal, Indoor, and Mobile Radio Communication (PIMRC), Washington DC, 2-5 September 2014, 1259-1263. https://doi.org/10.1109/PIMRC.2014.7136361

[15] Krishnaiah, R.V. and Surthineni. Ashok (2013) Overview and Evaluation of Bluetooth Low Energy: An Emerging Low-Power Wireless Technology. International Journal of Advanced Research in Computer Science and Software Engineering (IJARCSSE), 9, 736-740.

[16] Gomez, C., Oller, J. and Paradells, J. (2012) Overview and Evaluation of Bluetooth Low Energy: An Emerging Low-Power Wireless Technology. Sensors (Switzerland), 12, 11734-11753. https://doi.org/10.3390/s120911734

[17] Balogh, A. and Imre, S. (2017) Simulation and Analysis of Concurrent BLE Link Layer State Machines Running within the Same Physical Device. 25th International Conference on Software, Telecommunications and Computer Networks, Split, 21-23 September 2017, 1-8. https://doi.org/10.23919/SOFTCOM.2017.8115512

[18] OMNET++ (2019). http://www.omnetpp.org 